\title{
PRÁTICAS INOVADORAS DE EDUCAÇÃO EM SAÚDE BUCAL PARA PROMOÇÃO DA SAÚDE: RELATO DE EXPERIÊNCIA
}

\author{
Daniela Lemos Carcereri \\ Universidade Federal de Santa Catarina \\ daniela.lemos.carcereri@ufsc.br \\ Ana Carolina Oliveira Peres \\ Universidade Federal de Santa Catarina \\ anacaroll.peres@gmail.com \\ Caroline Paula Ludwig \\ Universidade Federal de Santa Catarina \\ carolinelud@gmail.com
}

Thayná Ferreira Simões Oliveira Universidade Federal de Santa Catarina thaynasimoes@hotmail.com

Alana Gabriele Menosso Universidade Federal de Santa Catarina alanamenosso@hotmail.com

Julia Quadri Bortoli Universidade Federal de Santa Catarina juliabortoli@hotmail.com.br

Renata Marques da Silva Universidade Federal de Santa Catarina odontologiarenata@gmail.com

Resumo

A Política Nacional de Promoção da Saúde (PNPS) aponta estratégias para produzir saúde sendo a escola um espaço privilegiado para o desenvolvimento destas ações. Este artigo objetiva relatar as experiências durante as práticas educativas em saúde bucal desenvolvidas por graduandos e pós-graduandos de Odontologia em um projeto de extensão (PE) voltado aos escolares do ensino fundamental. O PE destacou-se ao adotar o referencial da PNPS, a metodologia Freiriana, superando o modelo tradicional da educação em saúde bucal, favoreceu o planejamento, execução e avaliação das ações, desenvolveu competências necessárias ao futuro dentista a partir do tripé ensino, pesquisa e extensão na formação odontológica. Concluiu-se que esta vivência dos acadêmicos colaborou com a formação generalista e interdisciplinar, o vínculo, a troca de saberes e inovou nas práticas de educação em saúde articuladas com as temáticas prioritárias da PNPS.

Palavras-chaves: Saúde Bucal. Ensino em Saúde. Extensão Comunitária.

\section{INNOVATIVE PRACTICES IN ORAL HEALTH EDUCATION FOR HEALTH PROMOTION: EXPERIENCE REPORT}

Abstract

The Brazilian Health Promotion Policy (PNPS) points strategies to produce health, been the school, the school as a privileged space to developed these actions. This article aims to report the experiences during oral health education actions developed by undergraduate and graduate students of dentistry in an extension project (EP) aimed at elementary school students. The EP stood out by adopting the PNPS referential, the Freire's methodology, surpassed the traditional model of oral health education, favored the planning, execution and evaluation of the actions, developed the necessary skills for the future dentist from the tripod teaching, research and extension in dental education. It was concluded that this academic experience favored general and interdisciplinary education, a real bond, exchange of knowledge and innovation in health education practices articulated with the priority themes of PNPS.

Key Words: Oral Health. Dental Education. Community-institutional Relations.

\section{PRÁCTICAS INNOVADORAS DE EDUCACIÓN EN SALUD BUCAL PARA PROMOCIÓN DE LA SALUD: RELATO DE EXPERIENCIA}

\section{Resumen}

La Política Nacional de Promoción de la Salud (PNPS) apunta estrategias para producir salud y la escuela es um espacio privilegiado para el desarrollo destas acciones. Este artículo tiene por objeto relatar las experiencias durante prácticas educativas en salud bucal desarrolladas por graduandos y postgraduandos de Odontología en un proyecto de extensión (PE) dirigido a los escolares de la enseñanza fundamental. El PE se destacó al adoptar el referencial de la PNPS, la metodología Freiriana, trasponiendo el modelo tradicional de la educación en salud bucal, favoreció la planificación, ejecución y evaluación de las acciones, desarrolló competencias necesarias para el futuro dentista a partir del trípode enseñanza, investigación y extensión en la formación odontológica. Se concluyó que esa vivencia de los académicos favoreció la formación generalista e interdisciplinaria, vínculo, intercambio de saberes e innovó en las prácticas de educación en salud articuladas con las temáticas prioritárias de la PNPS.

Palabras clave: Salud Bucal. Educación en Odontología. Relaciones Comunidad-Institución. 
Práticas inovadoras de educação em saúde bucal para promoção da saúde: relato de experiência

\section{INTRODUÇÃO}

Compreende-se a Promoção da Saúde como "um conjunto de estratégias e formas de produzir saúde, no âmbito individual e coletivo, caracterizando-se pela articulação e cooperação intra e intersetorial [...] buscando articular suas ações com as demais redes de proteção social, com ampla participação e controle social” (BRASIL, 2014).

A Política Nacional de Promoção da Saúde (PNPS), visa garantir a integralidade do cuidado no Sistema Único de Saúde (SUS) em busca de qualidade de vida (BRASIL, 2014). Dentre os territórios possíveis para desenvolvimento de ações de promoção da saúde, a escola destaca-se como um espaço privilegiado, visto que contribui na construção de valores pessoais, crenças, conceitos e formas de compreender a realidade, o que influencia na produção social da saúde. A saúde bucal é parte integrante e essencial da saúde geral, com base nas diretrizes da Política Nacional de Saúde Bucal (PNSB) se insere no Programa Saúde na Escola (PSE) por meio de desenvolvimento de ações de prevenção de doenças, promoção, educação e recuperação da saúde bucal (BRASIL, 2004; BRASIL 2009).

As ações de educação em saúde bucal historicamente encontram-se fundamentadas na transmissão dos conhecimentos, na comunicação unidirecional, dogmática, autoritária, com foco na doença e na cura (FREITAS; MANDÚ, 2010; SILVA; CARCERERI; AMANTE, 2017) tendo como principais estratégias as palestras, o teatro de fantoches, a escovação supervisionada com macro-modelos (PAULETO, et.al., 2004), caracterizando ações centradas no cirurgião-dentista que não encorajam os escolares a refletir, dialogar e a conquistar a autonomia do cuidado (SILVA; CARCERERI; AMANTE, 2017).

Para inovar nas práticas de educação em saúde bucal torna-se fundamental superar estes modelos por outros que efetivem ações voltadas para além da prevenção de agravos em saúde bucal (FREITAS; MANDÚ, 2010). Por ser uma atividade de prática essencialmente intersetorial e interdisciplinar, a educação em saúde requer uma construção coletiva e inovadora quer seja no referencial adotado, nas estratégias utilizadas ou nos recursos tecnológicos, deslocando assim o foco da doença para a promoção da saúde e a integralidade do cuidado (MACHADO, et.al., 2007).

A extensão é um dos pilares do tripé universitário que mais aproxima os estudantes da comunidade, tendo como objetivo principal estabelecer uma relação transformadora entre a universidade e a sociedade na qual está inserida. $\mathrm{Na}$ área da saúde, em especial, a extensão enriquece a formação acadêmica dos futuros profissionais e pode contribuir para 
Práticas inovadoras de educação em saúde bucal para promoção da saúde: relato de experiência

melhorar a qualidade de vida da população pela possibilidade de intervenção sobre os determinantes sociais (BUSS, 2000).

O Projeto de Extensão (PE) 'Promoção de saúde na comunidade escolar do Colégio Aplicação da UFSC" é realizado há mais de 10 anos no Colégio de Aplicação (CA) da Universidade Federal de Santa Catarina (UFSC) em Florianópolis-SC, tendo como pressupostos a promoção da saúde, a interdisciplinaridade e a formação de multiplicadores para o trabalho com a temática da saúde bucal.

Participam do projeto estudantes da $2^{\mathrm{a}}, 3^{\mathrm{a}}$, $9^{\mathrm{a}}$ e $10^{\mathrm{a}}$ fase do Curso de Graduação em Odontologia da UFSC e pós-graduandos da área de concentração Odontologia em Saúde Coletiva, do Programa de Pós-graduação em Odontologia da UFSC. Para os discentes das fases iniciais da Graduação em Odontologia, o PE aporta uma inserção precoce e relevante para a formação crítico-reflexiva de profissionais implicados com as práticas de promoção da saúde e que compreendam a importância da intersetorialidade. Para os graduandos das fases finais o PE amplia os campos de atuação teórico-práticos e para os pós-graduandos oportuniza a vivência no ensino, na pesquisa e na extensão, fundamentais para a formação de um futuro docente. O grupo de estudantes é apoiado por professores e gestores do CA e conta com supervisão de uma docente do Departamento de Odontologia/UFSC.

O PE tem como objetivo articular as temáticas prioritárias da PNPS com a saúde bucal de forma atrativa, por meio de ações educativas, com intuito de despertar nos escolares o exercício da cidadania desenvolvendo responsabilidade pessoal e social com o meio em que vivem (FEIJÃO; GALVÃO, 2007; COSTA, et.al, 2015).

O presente artigo objetiva divulgar ações inovadoras de educação em saúde bucal, a partir do relato de experiência de um PE. A seguir serão descritas as atividades desenvolvidas, os resultados obtidos, apresentando-se as conclusões segundo a perspectiva de um grupo de estudantes de Odontologia, atuando sob supervisão docente.

\section{MATERIAIS E MÉTODOS}

O PE destina-se à promoção de saúde, com ênfase em ações de saúde bucal voltadas aos estudantes do ensino fundamental de primeiro ao nono ano, totalizando 27 turmas e aproximadamente 800 escolares. Fazem parte do escopo de ações do PE atividades de educação em saúde bucal, realização de levantamento epidemiológico em saúde bucal, oferta de atendimento clínico odontológico. 
Práticas inovadoras de educação em saúde bucal para promoção da saúde: relato de experiência

No segundo semestre de 2016, após a realização da territorialização na comunidade escolar pelos graduandos do PE, realizaram-se reuniões interdisciplinares de planejamento das ações de educação em saúde bucal entre os integrantes do PE e os docentes do CA. Nessas reuniões, foram discutidas as demandas da escola e as potencialidades do PE. Definiram-se as temáticas que necessitavam de uma abordagem conjunta: conceito ampliado de saúde, alimentação saudável, hábitos de higiene e sustentabilidade.

Planejou-se um cronograma de atividades que foram desenvolvidas ao longo do segundo semestre escolar de 2016 e primeiro trimestre de 2017, abrangendo alunos de $1^{\circ}$ ao $5^{\circ}$ ano do ensino fundamental, totalizando 15 turmas com 500 escolares. As atividades foram desenvolvidas às terças-feiras pela manhã nas turmas de $4^{\circ}$ e $5^{\circ}$ anos e nas quintasfeiras à tarde de $1^{\circ}$ ao $3^{\circ}$ ano.

Para trabalhar o tema de conceito ampliado em saúde, os graduandos do PE em parceria com uma professora de Educação Física do CA, elaboraram um circuito de atividades físicas associadas com perguntas de saúde bucal. As atividades foram desenvolvidas no pátio do CA, orientados pelas graduandas participantes do PE e um pósgraduando supervisionando a ação juntamente com a professora de Educação Física. Após a oficina, já em sala de aula, foram feitos exercícios de fixação sobre as temáticas abordadas e refletiu-se sobre o conceito ampliado de saúde.

A temática de alimentação saudável foi abordada com a oficina " Quanto açúcar? ”, elaborada pelas graduandas participantes do PE. O objetivo da oficina foi refletir sobre o consumo excessivo de açúcar em produtos industrializados, por meio de atividades lúdicopedagógicas voltadas para a leitura de rótulos e um comparativo visual da quantidade de açúcar oculto nos diferentes produtos usualmente consumidos. Com isso, foi possível avaliar o impacto negativo do excesso de açúcar não só no que diz respeito à doença cárie, mas também à diabetes e obesidade. Além disso, refletiu-se junto com os alunos do CA sobre as propagandas de produtos industrializados e as vantagens da alimentação natural.

O CA, desde 2015, trabalha com o projeto Lixo Zero e temáticas de sustentabilidade. Nesse contexto, emergiu nas discussões entre o PE e os docentes do CA a necessidade do uso racional de recursos como a água e o papel toalha nos banheiros da escola. Os graduandos participantes do PE elaboraram uma oficina que relacionou hábitos de higiene e o desperdício de recursos. Na oficina, o hábito de escovação dentária foi orientado, salientando o uso consciente da água, do uso excessivo e desnecessário de creme dental e do tempo de uso de uma escova de dente. Também se explicou a técnica correta de lavagem de mãos sendo que, por meio de atividade lúdica realizada no banheiro, os 
Práticas inovadoras de educação em saúde bucal para promoção da saúde: relato de experiência

alunos puderam avaliar a quantidade de papel toalha necessários para secar as mãos, evitando desperdício de recursos financeiros e do meio ambiente.

Ao final de cada atividade executada eram feitas avaliações com os estudantes do CA por meio de uma conversa. Já com os participantes do PE, eram feitas reuniões entre os graduandos e pós-graduandos para avaliar coletivamente as oficinas, ajustando-as, caso necessário, para atingir os objetivos propostos.

\section{RESULTADOS E ANÁLISE}

É importante destacar a relevância do PE ser comprometido com a transformação das práticas institucionais, pois ao adotar o referencial da promoção da saúde e a metodologia Freiriana da educação popular em saúde, inova e supera a tradição da prática focada apenas na prevenção da cárie dentária. Ademais, ao ampliar e integrar a saúde bucal de forma transversal no cotidiano escolar, proporcionou uma vivência em saúde bucal diferenciada.

Ao trabalhar com base nesse enfoque, foi possível observar que os participantes inovaram nas suas relações ao interagir com escolares e professores, reconstruindo a prática educativa; inovaram sua prática pedagógica com novas atitudes, reconstruindo velhas formas de pensar e fazer saúde. O PE contribuiu também para estimular a consciência crítica e o exercício da autonomia frente às decisões de saúde no âmbito individual e coletivo e, principalmente, inovou o rol de ações disponíveis para o processo de trabalho, facilitando a construção e implementação de uma comunidade promotora de saúde (ROCHA, et. al., 2002; FREIRE, 2006; FERREIRA, et.al., 2009).

A aproximação do graduando de odontologia com a realidade socioeducativa dos estudantes do CA favoreceu o planejamento de ações baseadas na compreensão da realidade e identificação das necessidades da comunidade escolar, articulando os contextos de saúde e educação. O processo de aprendizagem deve ter como ponto de partida a reflexão sobre o que acontece no serviço e o que precisa ser transformado. Para isto, faz-se necessário problematizar as situações cotidianas e trabalhar a partir da aprendizagem significativa (que promove e produz sentidos). Esse processo de educação no trabalho objetiva garantir a qualidade da atenção à saúde aproximando o educando das reais necessidades da população (PAGANI; ANDRADE, 2012).

O processo de planejamento, execução e avaliação das oficinas de educação em saúde bucal propiciaram aos graduandos desenvolver competências pessoais e 
Práticas inovadoras de educação em saúde bucal para promoção da saúde: relato de experiência

profissionais, necessárias para um futuro cirurgião-dentista. Ao longo do processo, os integrantes do PE trabalharam na perspectiva de uma comunicação emancipatória com os atores envolvidos, desenvolvendo reflexões éticas, criticidade, habilidades de comunicação, pró-atividade na construção de práticas educativas, autonomia e a criatividade em situações complexas. O contato direto e precoce do acadêmico com o mundo real por meio de projetos de extensão é fundamental para a sua formação, permitindo aplicar os conhecimentos adquiridos em sala de aula no cotidiano (HENNINGTON, 2005).

Um aspecto importante das oficinas elaboradas pelo PE foi que a demanda das temáticas abordadas foi pactuada de maneira conjunta com os docentes e discentes do CA. Além disso, nos momentos de avaliação, ao final das atividades, as falas dos alunos do CA foram valorizadas e direcionaram as mudanças necessárias, assim como destacaram os aspectos que deviam ser mantidos nas atividades.

As oficinas trouxeram de maneira lúdica temas relevantes não só de saúde bucal, mas também de saúde ampliada e respeito ao meio ambiente, adaptadas às diferentes faixas etárias (alunos entre 6 a 11 anos). Outro impacto positivo observado foi a criação de vínculo entre os integrantes do PE com os discentes e docente do CA.

A questão de hábitos de higiene e sustentabilidade, abordada por uma das oficinas, foi complementar às demais iniciativas já realizadas na escola com o Projeto Lixo Zero, na tentativa de conscientizar os alunos a evitarem desperdício de recursos financeiros e da natureza, como por exemplo papel toalha.

Percebeu-se uma maior expectativa para participarem da oficina que trabalhou o conceito ampliado de saúde com práticas corporais e desafios referentes à saúde bucal.

$\mathrm{Na}$ oficina que abordou a alimentação saudável, a partir da reflexão sobre a quantidade de açúcar nos produtos industrializados usualmente consumidos, foi possível notar a surpresa dos alunos com as informações trazidas na forma de um jogo.

A atividade lúdica é entendida como um mediador no processo ensinoaprendizagem. O lúdico chama a atenção para um assunto específico, o significado pode ser discutido entre todos os participantes e o produto da atividade lúdica diz respeito ao conhecimento que pode ser transportado para o campo da realidade (COSCRATO; PINA; MELLO, 2010).

Os graduandos das fases iniciais foram motivados e divulgaram as atividades desenvolvidas no PE em eventos científicos e semanas acadêmicas do curso, demonstrando a potencialidade do tripé ensino, pesquisa e extensão na formação odontológica. 
Práticas inovadoras de educação em saúde bucal para promoção da saúde: relato de experiência

Devido à dinâmica e a quantidade de crianças por atividade, não foi possível adaptar e realizar as oficinas com os escolares de $6^{\circ}$ ao $9^{\circ}$ ano, tornando-se assim um ponto a ser melhorado nas próximas edições do PE.

\section{CONSIDERAÇÕES FINAIS}

A oportunidade de atuação interdisciplinar nesse projeto de extensão inovou ao adotar os referenciais da promoção da saúde e da educação popular em saúde junto à comunidade escolar, possibilitou aos graduandos e pós-graduandos a ampliação do processo crítico-reflexivo, estimulou a pró-atividade, autonomia, análise e aplicação de conhecimentos técnicos referentes às temáticas de saúde bucal provenientes do processo de pesquisa para elaboração do PE e do aprendizado em sala de aula.

O projeto também se mostrou potente e fundamental para a formação de um futuro profissional generalista, articulado com as necessidades da população capaz de atuar em diferentes espaços sociais, realizando ações de promoção, prevenção e recuperação da saúde bucal.

A realização das oficinas de educação em saúde, com temáticas pactuadas entre os integrantes do PE e docentes do CA, contribuiu para a criatividade na elaboração das práticas por meio da troca de saberes entre os diferentes atores, trazendo uma abordagem inovadora e promotora de saúde, que ao utilizar o lúdico favoreceu o aprendizado das crianças gerando assim um impacto positivo. Portanto, um dos méritos dessa experiência está, justamente, no método inovador que admitiu uma prática pedagógica ética, crítica, reflexiva e transformadora, que superou os limites do ensinamento puramente técnicoodontológico.

O projeto seguirá acompanhando a condição de saúde da comunidade escolar, adaptando-se às transformações e necessidades percebidas pelos educandos. Expandir os horários e dar mais voz aos pais e aos escolares são objetivos de melhorias para as próximas edições do PE.

\section{AGRADECIMENTOS}

Ao Colégio de Aplicação da Universidade Federal de Santa Catarina por estar de portas abertas à formação profissional e apoiar o Projeto de Extensão 'Promoção de saúde na comunidade escolar do Colégio Aplicação da UFSC" há mais de uma década, 
Práticas inovadoras de educação em saúde bucal para promoção da saúde: relato de experiência

motivando práticas inovadoras e acreditando na construção coletiva de uma escola promotora de saúde.

Ao Departamento de Odontologia pelo suporte à sustentação do projeto.

À Pró-reitoria de Extensão da Universidade Federal de Santa Catarina pelo apoio e financiamento de uma bolsa de graduação por meio do Programa Pró-Bolsas 2017 e em edições anteriores.

\section{REFERÊNCIAS}

BRASIL. Ministério da Saúde. Portaria n 2.446, de 11 de novembro de 2014. Redefine a Política Nacional de Promoção da Saúde (PNPS). Disponível em: http://bvsms.saude.gov.br/bvs/saudelegis/gm/2014/prt2446 1111 2014.html. Acesso em: 29 mai. 2017.

BRASIL. Ministério da Saúde. Secretaria de Atenção à Saúde. Departamento de Atenção Básica. Coordenação Nacional de Saúde Bucal. Diretrizes da Política Nacional de Saúde Bucal - PNSB. Brasília; 2004. Disponível em: http://dab.saude.gov.br/portaldab/pnsb.php. Acesso em: 21 mai. 2017.

BRASIL. Ministério da Saúde. Secretaria de Atenção à Saúde. Departamento de Atenção Básica. Saúde na escola - Cadernos de Atenção básica n. 24. Brasília, 2009.

BUSS, Paulo Marchiori. Promoção da saúde e qualidade de vida. Ciência \& Saúde Coletiva, Rio de Janeiro, v. 5, n. 1, p.163-177, 2000.

COSCRATO, Gisele; PINA, Juliana Coelho; MELLO, Débora Falleiros de. Utilização de atividades lúdicas na educação em saúde: uma revisão integrativa da literatura. Acta Paulista de Enfermagem, São Paulo, v. 23, n. 2, p.257-263, abr. 2010.

COSTA, Deiziane Viana da Silva et al. Extensão universitária na promoção da saúde infantil: analisando estratégias educativas. Revista Ciência em Extensão, São Paulo, v. 11, n. 1, p.25-31, 2015.

FEIJÃO, Alexsandra Rodrigues; GALVÃO, Marli Teresinha Gimeniz. Ações de educação em saúde na atenção primária: revelando métodos, técnicas e bases teóricas. Revista da Rede de Enfermagem do Nordeste, Fortaleza, v. 2, n. 8, p.41-49, mai./ago. 2007.

FERREIRA, Maria de Lourdes da Silva Marques; COTTA, Rosângela Minardi Mitre; OLIVEIRA, Marilda Siriani de. Construção coletiva de experiências 
inovadoras no processo ensino-aprendizagem na formação de profissionais da saúde. Revista Brasileira de Educação Médica, Rio de Janeiro, v. 33, n. 2, p.240246, abr./jun. 2009.

FREIRE, Paulo. Pedagogia da autonomia: saberes necessários à prática educativa. 33. ed. São Paulo: Paz e Terra; 2006.

FREITAS, Maria de Lourdes de Assis; MANDÚ, Edir Nei Teixeira. Promoção da saúde na Estratégia Saúde da Família: análise de políticas de saúde brasileiras. Acta Paulista de Enfermagem, São Paulo, v. 23, n. 2, p.200-205, abr. 2010.

HENNINGTON, Élida Azevedo. Acolhimento como prática interdisciplinar num programa de extensão universitária. Cadernos de Saúde Pública, Rio de Janeiro, v. 21, n. 1, p.256-265, jan./fev., 2005.

MACHADO, Maria de Fátima Antero Sousa et al. Integralidade, formação de saúde, educação em saúde e as propostas do SUS: uma revisão conceitual. Ciência \& Saúde Coletiva, Rio de Janeiro, v. 12, n. 2, p.335-342, mar./abr. 2007.

PAGANI, Rosani; ANDRADE, Luiz Odorico Monteiro de. Preceptoria de território, novas práticas e saberes na estratégia de educação permanente em saúde da família: o estudo do caso de Sobral, CE. Saúde e Sociedade, São Paulo, v. 21, n. 1, p.94-106, mai. 2012.

PAULETO, Adriana Regina Colombo; PEREIRA, Maria Lucia Toralles; CYRINO, Eliana Goldfarb. Saúde bucal: uma revisão crítica sobre programações educativas para escolares. Ciência \& Saúde Coletiva, Rio de Janeiro, v. 9, n. 1, p.121-130, 2004.

ROCHA, Dais Gonçalves; MARCELO, Vânia Cristina; PEREIRA, Isabel M. T. Bicudo. Escola promotora da saúde: uma construção interdisciplinar e intersetorial. Journal Of Human Growth And Development, São Paulo, v. 12, n. 1, p.57-62, 2002.

SILVA, Grasiela Garret da; CARCERERI, Daniela Lemos; AMANTE, Cláudio José. Estudo qualitativo sobre um programa de educação em saúde bucal. Cadernos de Saúde Coletiva, Rio de Janeiro, v. 25, n. 1, p.7-13, 2017. 